\title{
Prospective randomized clinical trial evaluating the impact of vinegar on lipids in non-diabetics
}

\author{
Carmelo J. Panetta ${ }^{1,2 \#}$, Yvonne C. Jonk ${ }^{1}$, Alice C. Shapiro ${ }^{1,3}$ \\ ${ }^{1}$ University of Minnesota, Minneapolis, USA \\ ${ }^{2}$ HealthEast Heart Care, St. Paul, USA \\ ${ }^{3}$ Health Research, Park Nicollet Institute, Minneapolis, USA \\ Email: "panet002@umn.edu
}

Received 13 January 2013; revised 1 March 2013; accepted 8 April 2013

Copyright (C) 2013 Carmelo J. Panetta et al. This is an open access article distributed under the Creative Commons Attribution License, which permits unrestricted use, distribution, and reproduction in any medium, provided the original work is properly cited.

\begin{abstract}
Background: Heart disease is now considered an inflammatory process targeted primarily by medical therapy on lipid levels. Complementary and alternative medicine searches for novel non-pharmacologic therapy, including pursuing various diets. Animal studies and consumer literature suggest benefits of vinegar on lipid levels and diabetes mellitus. Our nonrandomized pilot study from our group suggested a benefit in raising high-density lipoprotein cholesterol (HDL-C). Based on this data, we conducted a randomized placebo controlled clinical trial to determine the effects of apple cider vinegar intake in those without diabetes mellitus on total cholesterol (TC), low-density lipoprotein cholesterol (LDL-C), triglycerides, HDL-C, Hemoglobin A1C (Hgb-A1C) and measurement of inflammation with high sensitivity CRP levels (HS-CRP). Methods: A prospective randomized, double blind, placebo-controlled clinical trial consisting of 114 participants was conducted. Participants consumed $30 \mathrm{~mL}$ of either apple cider vinegar or placebo for two months. Measurements were collected at baseline, eight and sixteen weeks. The primary endpoint was the change in HDL-C from baseline to eight weeks between the vinegar and placebo groups. Secondary endpoints were change from baseline to eight weeks in TC, LDL-C, triglycerides, Hgb-A1c and HS-CRP. Results: Change in serum HDL-C concentration was not significantly different between the vinegar and control groups after eight weeks of supplementation. Secondary end-

\footnotetext{
*Grant support: University of Minnesota, Lillehei Heart Institute and Park Nicollet Foundation provided grants for funding for the pilot and randomized trial.

No competing financial interests exist for the authors.

${ }^{\#}$ Corresponding author.
}

points including TC, LDL-C, Hgb-A1c and HS-CRP were not statistically different at the Bonferroni corrected significance level of 0.01 . No significant difference was found regardless of baseline HDL-C levels. Conclusions: We found no significant difference in HDL-C, LDL-C, triglycerides, total cholesterol, or HS-CRP levels with use of vinegar but a trend down of Hgb-A1c in this group of non-diabetic participants. Further investigation is required to define the impact of vinegar in those with diabetes mellitus.

Keywords: Vinegar; High-Density Lipoprotein; Low-Density Lipoprotein; High Sensitivity C-Reactive Protein; Triglycerides

\section{INTRODUCTION}

Heart Disease has been the leading cause of death in the United States, and a major cause of disability [1]. In 2009 , the prevalence of coronary heart disease, including heart attack, angina, and stroke, ranged from $2.5 \%$ to $10.3 \%$ across US states or territories [2]. Risk factors can include diabetes mellitus, smoking tobacco, age, family history, hypertension, male sex and hyperlipidemia. Among those with lipid abnormalities, increased low density lipoprotein cholesterol (LDL-C) and reduced highdensity lipoprotein cholesterol (HDL-C) concentrations have been associated with heart disease [3-7]. Conversely, higher HDL-C levels have been correlated with fewer cardiac events including those with low LDL-C [8].

The optimal pharmacologic treatment to increase HDL-C levels was prescribing Niacin; however, Niacin's success is limited by side effects and a recent report questioning it's clinical impact $[9,10]$. Novel forms of therapy which increase HDL-C have been studied but are 
not available to the general public [7,11-13]. Non-pharmacologic treatments including life style changes such as smoking cessation, weight loss, physical activity and alcohol consumption can increase HDL-C from $12 \%$ to $21 \%[7,14,15]$. Alcohol has been associated with lower risk for myocardial infarction, in part through impacting HDL-C [16-18]. Although a recent article challenges the theory based on no relationship to fatal coronary heart disease [19], nonfatal coronary heart disease is still in question [20]. Vinegar as a byproduct of alcohol, could serve as an alternative to alcohol on lipids.

The dietary intake of vinegar has been reported to lower triglyceride levels [21], reduce LDL-C, and raise HDL-C in several animal studies [22,23]. We performed a small nonrandomized pilot study with one, two, three or six teaspoons of apple cider vinegar and found an increase in HDL-C trended with increase in dose. Vinegar could be an adjunct to traditional pharmacologic treatments to reduce patients' risk of coronary heart disease. Complementary and alternative medicines are used by over two thirds of Americans [24] and provide an option for treatment if validated by randomized controlled clinical trials $[25,26]$. In order to assess whether vinegar effectively lowers patient's risk of coronary heart disease, randomized control trials are needed. To date, no randomized controlled trials have been conducted to clinically establish the benefits of vinegar on cholesterol.

The purpose of this study was to rigorously address whether the dietary intake of vinegar increases HDL-C levels. Secondary objectives were to address whether increasing dosages of vinegar had a positive effect on reducing risk factors, namely HDL-C levels, as well as triglycerides, LDL-C, Hgb-A1c and HS-CRP.

\section{METHODS}

We conducted a randomized double blind placebo controlled trial looking at the effects of varying dietary dosages of vinegar on HDL-C. A convenience sample of patients who visited a mid western urban specialty (cardiac) clinic and/or who responded to recruitment information (flyers/internet website) within the clinic's integrated provider system participated in the study. Those with diabetes mellitus were excluded from the trial due to one prospective study in adults with diabetes mellitus found vinegar improved insulin sensitivity [27]. HgbAlc was measured in the event some of the participants had undiagnosed diabetes mellitus. Other exclusion criteria included current tobacco use, alcohol consumption greater than 2 drinks per day, use of niacin pharmaceutical products, and initiation or an increase in fibrates, ezetimibe, or statin therapy within 6 weeks randomization.

Participants were randomized equally to the treatment and control conditions. The control group supplemented their usual intake with $1.7 \%$ balsamic vinegar solution diluted in water and the treatment group with $100 \%$ apple cider vinegar (Bragg Apple Cider Vinegar with the mother, acetic bacteria, Live Food Products, Santa Barbara, CA). Both treatment and control solutions were prepared for the study duration in $500 \mathrm{~mL}$ brown glass bottles and color-coded to maintain a double-blind study. Neither the participants nor the researchers were aware of group assignment. The solution for Control Group was pre-tested to give a similar taste and odor as the experimental solution. Randomization was performed by a blinded coordinator who removed labels from an envelope, which created a list for the research coordinator to assign participants to either red or green labeled bottles. Study participants were instructed to take $30 \mathrm{~mL}$ (approximately 2 Tablespoons) per day of solution. The solution could be diluted with 16 ounces or less of juice or water, but not mixed with salads or other food to avoid incomplete intake. Due to a possible change in chemical composition, the solutions could not be boiled or placed in a hot solution (such as hot tea or apple cider) prior to drinking. Lipid analysis and HS-CRP were measured on ADVIA 1650 Chemistry System (Siemens Healthcare Diagnostics, Deerfield, IL). Hgb-A1c was measured on Variant Program (Bio-Rad Laboratories, Hercules, CA). Participants recorded their intake of daily solution and documented concerns or side effects in daily diaries. Alcohol intake data was collected and recorded daily over the 8-week intervention. Study subjects were issued pedometers and recorded their total number of steps per day. Samples were collected for HDL-C, TC, LDL-C, HgbA1c and HS-CRP following an overnight fast at baseline, two months and four months. Following the two-month visit, participants were instructed to discontinue their solutions and diaries, diaries and bottles were collected and participants were instructed to return for a final study visit for the four-month measurements.

\subsection{Sample Size}

Sample and effect sizes were based on the results of completing a small, prospective, non-randomized, pilot study evaluating the effect of escalating doses of apple cider vinegar on lipids. The pilot study found a $17 \%$ increase in the HDL-C after 8 weeks of two tablespoons daily of vinegar, similar to the impact of alcohol. Since the nonrandomized pilot study did not include a control group, we took a conservative approach in estimating effect size by considering the group receiving the lowest dosage of apple cider vinegar (1 tsp./day) in the small prospective pilot as our control group in the current study. Since the average change from baseline to two months for those patients taking 1 teaspoons of apple cider vinegar per day was $4 \mathrm{mg} / \mathrm{dl}$, and the average change in the group taking 2 tablespoons of apple cider vinegar per day 
was $7.8 \mathrm{mg} / \mathrm{dl}$, we hypothesized that the mean change of HDL-C was greater than $3.8 \mathrm{mg} / \mathrm{dl}$ when taking two tablespoons of apple cider vinegar per day. A sample size of 114 participants was computed for detecting a difference of $3.8 \mathrm{mg} / \mathrm{dL}$ with $80 \%$ power. This sample size included extra participants to account for a potential $15 \%$ drop out rate due to taste or other unknown factors.

\subsection{Statistical Analysis}

The mean change from baseline to two months, and baseline to four months for HDL-C, TC, LDL-C, Hb-A1c and HS-CRP were evaluated using paired t-tests within treatment group and independent t-tests between treatment groups. The effect of apple cider vinegar versus placebo on HDL-C, TC, LDL-C, triglycerides, Hgb-A1c and HS-CRP was also estimated using analysis of covariance (ANCOVA) while controlling for baseline HDL-C, age, sex, and body-mass index (BMI). Results for the primary endpoint, change in HDL-C, were considered statistically significant at the 0.05 level. Results for the secondary endpoints were considered statistically significant at the Bonferroni corrected significance level of 0.01. Baseline values between the control and experimental group were compared using independent t-tests for continuous variables and chi-square tests for dichotomous data.

Exploratory and subgroup analyses were conducted for different age groups, baseline HDL-C levels, alcohol use, daily step totals, and medication use. Results from these analyses were in agreement with the primary ana- lyses.

Statistical analyses were performed using SAS software (Statistical Analysis System, version 9.2: SAS Institute Inc., Cary, NC). Institutional Review Board approval was granted for this study. All patients signed informed consent prior to study participation.

\section{RESULTS}

One hundred and fourteen subjects were enrolled in the study, seventeen patients (15\%) dropped out, with taste intolerance accounting for seven (6\%) (Figure 1).

Baseline characteristics for the control and treatment groups are described in Table 1.

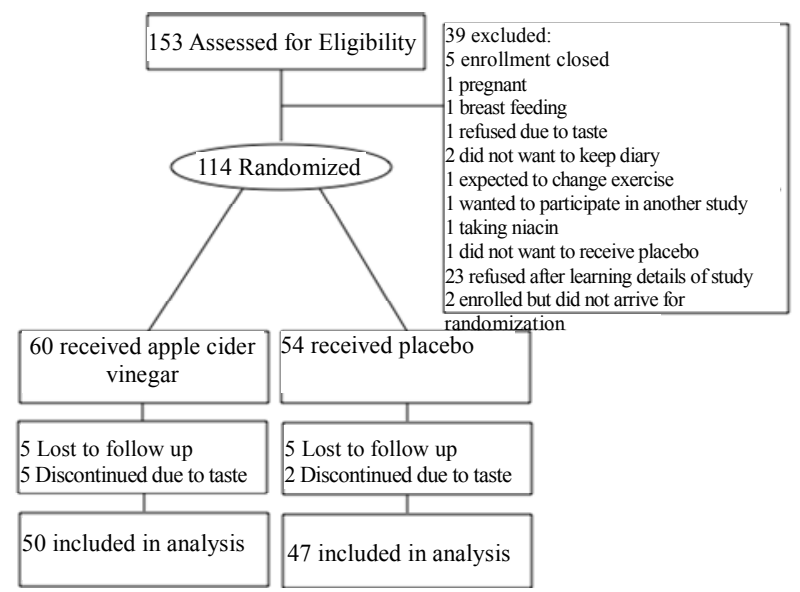

Figure 1. Flow diagram of randomization into apple cider vinegar treatment and control groups.

Table 1. Baseline characteristics.

\begin{tabular}{lccc}
\hline & Vinegar Treatment Group $(\mathrm{n}=50)$ & Control Group $(\mathrm{n}=47)$ & P-Value \\
\hline Characteristic & Mean (SD) & Mean $(\mathrm{SD})$ & \\
Age (years) & $57.7(9.33)$ & $56.1(12.58)$ & 0.50 \\
Weight $(\mathrm{kg})$ & $81.2(17.61)$ & $76.4(13.8)$ & 0.14 \\
BMI $\left(\mathrm{kg} / \mathrm{m}^{2}\right)$ & $28.3(5.87)$ & $26.8(4.19)$ & 0.17 \\
Total Cholesterol (mg/dL) & $190.3(40.69)$ & $192(42.84)$ & 0.84 \\
LDL-C $(\mathrm{mg} / \mathrm{dL})$ & $116.1(31.36)$ & $116(28.97)$ & 0.67 \\
HDL-C $(\mathrm{mg} / \mathrm{dL})$ & $52.5(14.16)$ & $54(19)$ & 0.99 \\
Triglycerides $(\mathrm{mg} / \mathrm{dL})$ & $108.8(59.97)$ & $109.4(62.62)$ & 0.96 \\
Hgb-Alc $(\%)$ & $5.7(0.38)$ & $5.7(0.43)$ & 0.79 \\
HS-CRP $(\mathrm{mg} / \mathrm{L})$ & $1.8(3.36)$ & $2.1(2.93)$ & 0.73 \\
& No. $(\%)$ & No. $(\%)$ & \\
Female & $33(0.66)$ & $30(0.64)$ & 0.84 \\
White & $43(0.86)$ & $39(0.83)$ & 0.78 \\
Statin & $21(0.42)$ & $14(0.3)$ & 0.29 \\
Fish oil & $21(0.42)$ & $17(0.36)$ & 0.68 \\
Ezetimibe & $2(0.04)$ & $4(0.09)$ & 0.43 \\
Fibrate & $1(0.02)$ & $6(0.13)$ & 0.05 \\
Beta-blocker & $9(0.18)$ & $14(0.3)$ & 0.23 \\
Aspirin & $24(0.48)$ & $14(0.3)$ & 0.10 \\
Ace inhibitor & $7(0.14)$ & $6(0.13)$ & 1.00 \\
\hline
\end{tabular}


The majority of participants were female $(65 \%)$ and Caucasian (87\%). The mean age was 56.7 years and the mean BMI was $28.4 \mathrm{~kg} / \mathrm{m}^{2}$. Approximately one third of participants were using statins $(36 \%)$ and/or fish oil (39\%). The control group had more participants on fibrates at baseline and throughout the study compared to the treatment (vinegar) group $(p=0.05)$. No differences were found in baseline lipids, Hgb-A1c, or HS-CRP. Dropout bias was not present when evaluating treatment group, age, gender, and baseline HDL-C using logistic regression. Adherence to the study solution was $96 \%$ for the cider vinegar group and $98 \%$ for the control group.

No significant differences were found in HDL-C levels between baseline and 8 weeks and baseline and 16 weeks in the vinegar group (Figure 2).

There was no significant difference in cholesterol levels from baseline to 8 weeks in the vinegar or placebo arm (Table 2). Noted a trend towards lower Hgb A1C was seen in the vinegar arm when comparing baseline to 8 weeks. The mean differences between the apple cider vinegar and control groups for change from baseline to week 8 for total cholesterol, LDL, HDL, triglycerides, $\mathrm{Hgb}-\mathrm{A} 1 \mathrm{c}$ and HS-CRP.

On further analysis, a marginally significant change in HDL-C was observed from the week 8 to week 16 visit between the treatment and control group after an 8 week wash-out period (mean difference $-2.50 \mathrm{mg} / \mathrm{dL}, 95 \% \mathrm{CI}$ $[-4.70,-0.30], \mathrm{p}=0.03)$. This difference in HDL-C was due to an increase of $1.50 \mathrm{mg} / \mathrm{dl}(95 \%$ CI $[-0.13,3.15])$ for the control group and a decrease of $0.98 \mathrm{mg} / \mathrm{dl}(95 \%$ CI $[-2.51,0.55])$ in the treatment group from week 8 to week 16. Yet, there was no significant change in HDL-C between the two groups from baseline to week 8 or week 16. HS-CRP was not impacted by vinegar, but a significant decline in HS-CRP in the control arm at 8 weeks was primarily responsible for the significant difference between the vinegar arm and the control arm at 8 weeks.

Although no exclusion was placed on the baseline HDL-C level, a subgroup analysis focusing on those with baseline HDL-C levels of less than $50 \mathrm{mg} / \mathrm{dL}(\mathrm{n}=48)$ did not provide evidence of a treatment effect from base- line to two months due to vinegar versus control (mean difference: $1.33 \mathrm{mg} / \mathrm{dl}, 95 \%$ CI [-2.06, 2.62]).

ANCOVA showed no statistically significant effect on HDL-C between treatment groups from baseline to two months for age $(p=0.14)$, sex $(p=0.20)$, body mass index $(p=0.60)$, or fibrate usage $(p=0.90)$. In addition, no statistically significant effect on HDL-C was found for the treatment when controlling for age, sex, body mass index, and fibrate usage $(p=0.13)$. Regression analyses for all secondary endpoints yielded non-significant results for treatment when controlling for age, sex, and BMI.

\section{DISCUSSION}

Complementary and alternative medicines are used by over two thirds of Americans [24] and may provide an option for treatment if validated by randomized controlled clinical trials. $[25,26]$ Vinegar as a byproduct of alcohol, would appear an alternative to alcohol for raising HDL-C. Vinegar has been used for a variety of aliments for centuries. Dr. James Lind in 1746 compared vinegar, dilute sulfuric acid, cider, sea water, a physician's remedy of nutmeg, garlic and horseradish mixture versus a diet of oranges and lemons to prevent scurvy

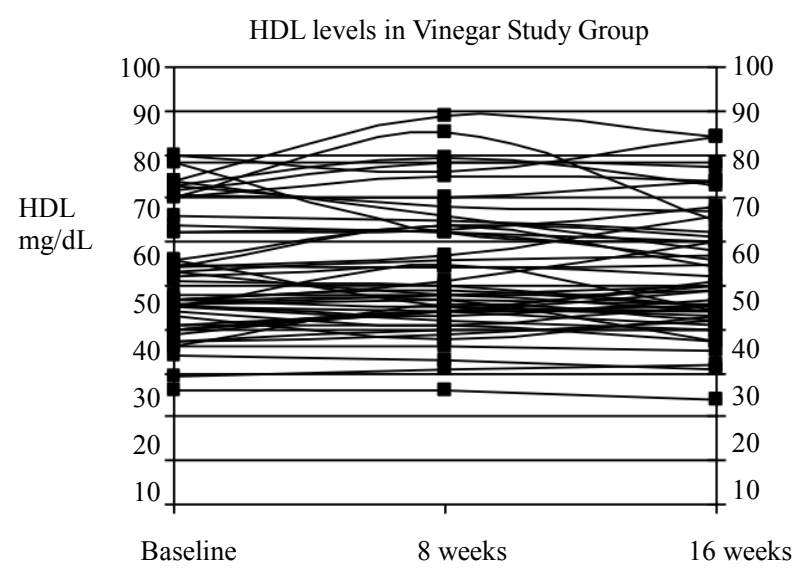

Figure 2. HDL-C levels at baseline compared to 8 and 16 weeks with apple cider vinegar treatment and control groups.

Table 2. Laboratory values from baseline to eight weeks of vinegar or placebo.

\begin{tabular}{ccccc}
\hline & \multicolumn{2}{c}{ Apple Cider Vinegar $(\mathrm{n}=50)$} & \multicolumn{1}{c}{ Placebo $(\mathrm{n}=47)$} \\
\hline Total Cholesterol (md/dL) & $\underline{\text { Mean }[95 \% \mathrm{CI}]}$ & $\underline{\text { P-Value }}$ & $\underline{\text { Mean }[95 \% \mathrm{CI}]}$ & $\underline{\mathrm{P}-\text { Value }}$ \\
LDL-C (mg/dL) & $1.22[-5.48,7.92]$ & 0.72 & $-3.02[-8.13,2.08]$ & 0.24 \\
HDL-C (mg/dL) & $-1.36[-6.99,4.27]$ & 0.63 & $-2.15[-6.02,1.72]$ & 0.27 \\
Triglycerides (mg/dL) & $1.14[-0.47,2.75]$ & 0.16 & $-1.06[-3.05,0.92]$ & 0.29 \\
Hgb-A1C (\%) & $5.98[-6.92,18.88]$ & 0.36 & $1.47[-10.02,12.96]$ & 0.80 \\
HS-CRP (mg/L) & $-0.07[-0.15,0.01]$ & 0.08 & $-0.01[-0.1,0.07]$ & 0.74 \\
\hline
\end{tabular}

Note: T-test was used to analyze mean change from baseline to 8 weeks. 
[28]. Recent data in animals suggest vinegar improves lipids, and our small non randomized trial implied improvement in HDL-C [21-23].

Unfortunately, our larger randomized trial results did not indicate that apple cider vinegar impacted HDL-C compared to control. Subgroup and other exploratory analyses were carried out by sex, age, medication, alcohol use, and steps data, but no significant change in HDL-C was observed. In addition, no evidence of vinegar impacting secondary endpoints including LDL-C, triglycerides, total cholesterol, and HS-CRP compared to a control group.

Persons with diabetes mellitus were intentionally excluded due to possible improvement in insulin resistance based on a single center prospective clinical study [27]. However, although not significant, there was an observed trend down in Hgb-A1c in persons without diabetes mellitus with vinegar treatment.

Limitations of the study included a drop out rate of $15 \%$, although this was expected based on a pilot study and the sample size was adjusted accordingly. Second, no exclusion was placed on the baseline HDL-C level, implying that vinegar may raise HDL-C only in those with low levels. Although a subgroup analysis focusing on those with baseline HDL-C levels of less than 50 $\mathrm{mg} / \mathrm{dL}$, did not provide evidence for a treatment effect from baseline to two months due to vinegar versus control. Third, the sample size is relatively small and does not account for different races or sex to exclude the possibility of an impact of apple cider vinegar on lipids.

In conclusion, after performing a randomized double blind study, we did not find evidence to support the use of vinegar for management of low HDL-C, elevated triglycerides or LDL-C. In addition, we found no improvement in HS-CRP and a trend down on Hbg-A1C with vinegar. Since vinegar may impact lipids indirectly in those with diabetes mellitus by improving glucose control [27], future studies focusing on this rising population should be considered.

\section{ACKNOWLEDGEMENTS}

Authors wish to thank Jennifer Mattson and Jennifer O'Connell for coordination and administrative assistance and Elizabeth Bisinov, MD and Elizabeth Kind, RN, MS for their critical review of the manuscript.

\section{REFERENCES}

[1] Li, C., Balluz, L.S., Okoro, C.A., et al. (2011) Centers for disease control and prevention (CDC). Surveillance of certain health behaviors and conditions among states and selected local areas-Behavioral Risk Factor Surveillance System, United States, 2009. MMWR Surveillance Summaries, 60, 1-250.

[2] Heron, M., Hoyert, D.L., Murphy, S.L., et al. (2009)
Deaths: Final data for 2006. National Vital Statistics Reports, 57, 1-134.

[3] Gordon, T., Castelli, W.P., Hjortland, M.C., et al. (1977) High density lipoprotein as a protective factor against coronary heart disease: The Framingham study. American Journal of Medicine, 62, 707-714. doi:10.1016/0002-9343(77)90874-9

[4] Gorden, D.J., Probstfield, J.L., Garrison, R.J., et al. (1989) High-density lipoprotein cholesterol and cardiovascular disease: Four prospective American studies. Circulation, 79, 8-15. doi:10.1161/01.CIR.79.1.8

[5] Assmann, G. and Schults, H. (1990) Modelling the Helsinki heart study by means of risk equations obtained from the PROCAM study and the Framingham heart study. Drugs, 40, 13-18. doi:10.2165/00003495-199000401-00005

[6] Toth, P. (2004) High-density lipoprotein and Cardiovascular risk. Circulation, 109, 1809-1812. doi:10.1161/01.CIR.0000126889.97626.B8

[7] Singh, I.M., Shishehbor, M.H. and Ansell, B.J. (2007) High-density lipoprotein as a therapeutic target. A Systemic Review. JAMA, 298, 786-798. doi:10.1001/jama.298.7.786

[8] Barter, P., Gotto, A.M., LaRosa, J.C., et al. (2007) HDL cholesterol, very low levels of LDL cholesterol, and Cardiovascular events. New England Journal of Medicine, 357, 1301-1310. doi:10.1056/NEJMoa064278

[9] Carlson, L.A. (2006) Nicotinic acid and other therapies for raising high-density lipoprotein. Current Opinion in Cardiology, 21, 336.

[10] http://www.nih.gov/news/health/may2011/nhlbi-26.htm

[11] Nissen, S.E., Tsunoda, T., Tuzcu, M. et al. (2003) Effect of recombinany ApoA-I Milano on coronary atherosclerosis in patients with acute coronary syndromes: A randomized controlled trial. JAMA, 290, 2292-2300. doi:10.1001/jama.290.17.2292

[12] Tardif, J.C., Gregoir, J., L'Allier, P.L., et al. (2007) Effects of reconstituted high-density lipoprotein infusions on coronary atherosclerosis. A randomized controlled trial. JAMA, 297, 1675-1682. doi:10.1001/jama.297.15.jpc70004

[13] Brousseau, M.E., Schafer, E.J., Wolfe, M.L., et al. (2004) Effects of an inhibitor of cholesteryl ester transfer protein on HDL cholesterol. New England Journal of Medicine, 350, 1505-1515. doi:10.1056/NEJMoa031766

[14] Van der Gaag, M.S., van Tol, A., Vermunt, S.H.F., et al. (2001) Alchohol consumption stimulates early steps in reverse cholesterol transport. Journal of Lipid Research, 42, 2077.

[15] Hartung, G.H., Foreyt, J.P., Mitchell, R., et al. (1983) Effect of alcohol intake on high-density lipoprotein cholesterol levels in runners and inactive men. JAMA, 249, 747-750. doi:10.1001/jama.1983.03330300031029

[16] Castelli, W.P., Doyle, J.T., Gordon, T. et al. (1977) Alcohol and blood lipids. The cooperative lipoprotein phenotyping study. Lancet, 2, 153-155. doi:10.1016/S0140-6736(77)90176-3

[17] Mukamal, K.J., Jensen, M.K., Gronbaek, M., et al. (2005) 
Drinking frequency, mediating biomarkers, and risk of myocardial infarction in women and men. Circulation, 112, 1406-1413. doi:10.1161/CIRCULATIONAHA.105.537704

[18] Linn, S., Carroll, M., Johnson, C., et al. (1993) Highdensity lipoprotein cholesterol and alcohol consumption in US white and black adults: Data from NHANES II. American Journal of Public Health, 83, 811-816. doi:10.2105/AJPH.83.6.811

[19] Magnus, R., Bakke, E., Hoff, D. et al. (2011) Controlling for high-density lipoprotein cholesterol does not affect the magnitude of the relationship between alcohol and coronary heart disease. Circulation, 124, 2296-2302. doi:10.1161/CIRCULATIONAHA.111.036491

[20] Krauss, R. and Williams, P. (2011) Alcholol and the heart: Does raising high-density lipoprotein matter? Circulation, 124, 2283-2284. doi:10.1161/CIRCULATIONAHA.111.067223

[21] Moon, Y.-J. and Cha, Y.-S. (2008) Effects of persimmonvinegar on lipid metabolism and alcohol clearance in chronic alcohol-fed rats. Journal of Medicinal Food, 11, 38-45. doi:10.1089/jmf.2007.071

[22] Sishehbor, F., Mansoori, A., Sarkaki, A.R., et al. (2008) Apple cider vinegar attenuates lipid profile in normal and diabetic rats. Pakistan Journal of Biological Sciences, 11,
2634-2638. doi:10.3923/pjbs.2008.2634.2638

[23] Setorki, M., Asgary, S., Eidi, A., et al. (2010) Acute effects of vinegar intake on some biochemical risk facgors of atherosclerosis in hypercholesterolemic rabbits. Lipids in Health and Disease, 9, 10. doi:10.1186/1476-511X-9-10

[24] Barnes, P.M., Powell-Friner, E., McFann, K., et al. (2002) Complementary and alternative medicine use among adults: United States. Advanced Data, 343, 1-19.

[25] Voelker, R. (2005) IOM points to need for more research, regulation in alternative medicine. JAMA, 293, 11781180.

[26] Chan, E. (2008) Quality of efficacy research in complementary and alternative medicine. JAMA, 299, 2685-2686. doi:10.1001/jama.299.22.2685

[27] Johnston, C., Kim, C. and Buller, A. (2004) Vinegar improves insulin sensitivity to a high-carbohydrate meal in subjects with insulin resistance of type 2 diabetes. Diabetes Care, 27, 281-282. doi:10.2337/diacare.27.1.281

[28] Lind, J. (1753) A treatise of the scurvy in three parts. Containing an inquiry into the nature, causes and cure of that disease, together with a critical and chronological view of what has been published on the subject. A. Millar, London. 